\title{
DØ Cryogenic Auto Dialing Alarm system
}

\author{
Dan Markley \\ AUGUST 3, 1992
}

D0 Engineering Note 3740.510.EN-294

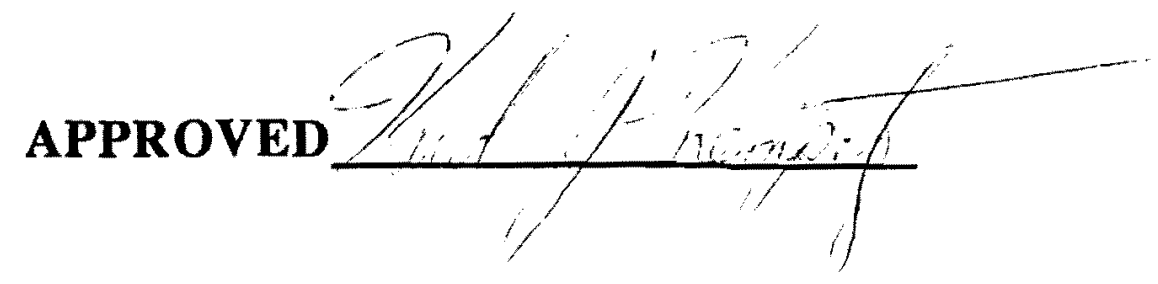




\section{DO KAYE AUTO DIALING ALARM SYSTEM}

Overview: The Automatic Dialing system purchased by DO is intended to help make the D0 cryogenic system operate unattended by cryogenic operating personnel. The auto dialer is completely programmable and is voice synthesized. The auto dialer was purchased with 32 bistable inputs, but is expandable to 64 bistable inputs with the purchase of more electronic cards at an approximate cost of $\$ 260$ per card( 8 bistable inputs). The auto dialer also has the capability for analog inputs, analog outputs, and bistable outputs none of which DO uses or intends to use. The auto dialer can be called on its' operating phone line to describe current alarms with the proper password. The Auto Dialer can dial lab extensions, lab pagers, and any number outside the lab. It cannot dial a long distance pager.

Operation: The auto dialer monitors alarms and alarm conditions via the TI565 PLC, upon an alarm condition it initiates a phone calling sequence of preprogrammed lists with assigned priorities. When someone is reached, the auto dialer describes the individual alarm it is calling for, by a preprogrammed set of words for that individual alarm, spoken by a female voice. The called person then has a chance to acknowledge the alarm over the telephone, if the alarm is not acknowledged the auto dialer will disconnect and call the next person on the list. The auto dialer will continue to cycle through the list until it is acknowledged, reset, or the alarm condition no longer exists.

\section{Specifications:}

Manufacturer:

Service:

Model:

Serial\#:

Power:

Fermilab extension\#
Kaye Instruments, Inc.

15 DeAngelo Drive

Bedford, MA 01730

617-275-0300

Ron Alphen $\times 283$

ADAS $3000, \times 7950$

006361

120 vac, $60 \mathrm{htz}, 1 \mathrm{amp}$

Internally Battery backed with charger $x 8065$ 


\section{Brogrammable Parameters:}

1. Individual alarm message( 300 word internal library).

2. 8 lists of 8 telephone numbers. The lower the list \#, the higher the priority.

3. The priority of each alarm channel, by assigning a list to it.

4. Open/closed input on alarm.

5. Each channel enable/disable.

6. Station ID phrase.

7. Incoming telephone ring count.

8. Real time clock.

9. Message repeat count.

10. Intercall Delay (allowed acknowledge time of an alarm).

11. Calling time of day.(time dialer is allowed to operate)

12. Call back acknowledge delay.(delay between outgoing alarm calls)

13. access code.

14. Bistable alarm delay.

15. Power fail call cancel.

16. Power fail time delay.

17. Power fail calling assignment.

Programming: Programming of the auto dialer is done through the keypad inside the front cover. The auto dialer is internally battery backed, therefore programmed instruction are never lost even when the unit is switched off. Since there are many programmable parameters and the keystroke commands are extensive, the owners manual should be consulted when reprogramming. The manual can be found in the DO cryogenic control room.

Alarm handling: Alarms are generated by the TI565 PLC. They can be of analog or digital nature. The analog and digital alarms are programmed into the ladder logic of the PLC. Both types of alarms are sent to the auto dialer by output isolated relay modules in the PLC I/O bases wired to the auto dialer inputs.

The auto dialer alarms are controlled from operator graphic pages of the DMACS control software. These pages are password protected. The digital auto dialer alarms can be enabled/disabled from this graphic page. The analog auto dialer alarms can be enabled/disabled and their threshold triggering points can be changed from this graphic page. 
Auto Dialer alarm acknowledging: When receiving an auto dialer alarm it can be dealt with in several ways.

1. Punch 8 on a touch tone phone after the message, to acknowledge.

2. Hang up call it back, enter the password, then acknowledge the alarm. Fermilab x8065

3. Push the reset button on the auto dialer. CAUTION: Doing this puts all of the auto dialer alarms in the bypass mode for the "bypass to run delay" time period. This should only be used temporarily to stop the outgoing calls from the auto dialer while you are assessing the alarm, because you will not be notified by the auto dialer while in this mode, if another auto dialer alarm is triggered. For longer term acknowledgment for a particular alarm, it should be disabled.

4. The individual alarm that has been triggered can be disabled, while assessing the alarm. This will disable the alarm to the auto dialer also.

Kaye Auto Dialer Weakness:_The Kaye Auto Dialing alarm system is designed to work on a touch tone dialing or a pulse dialing phone line. When it dials a number, the first digit is sent out as a tone. If there isn't a gap of a few milliseconds after the tone, the Auto Dialer assumes that the phone line is a pulse dialing line.

The Auto Dialer could not dial outside the Fermilab phone network because the Centrex phone system used is extremely fast. There is no gap in the dial tone after dialing a 9 to get an outside line. The Auto Dialer would then proceed to dial the number over using pulse dialing which would of course be the wrong number, since the first digit would be dialed twice. The Auto Dialer with a touch tone line, at Fermilab is limited to lab pagers and lab extensions.

This dialing problem was solved by having Illinois Bell change the phone line to a pulse dial only. Everytime the Auto Dialer dials a number it switches to pulse dialing after the first digit is dialed. Now that the first tone digit is invisible to the phone network, redialing of the number in pulse dialing works correctly.

The Auto Dialer can now dial lab pagers, lab extension, and any number outside the lab with a pulse dialing telephone line. The Auto Dialer cannot dial a long distance pager. The long distance pager requires a touch tone dialing system in order to work. 


\section{Currently Programmed Auto Dialer Messages:}

CH \# Voice Synthesized Message

1.

2. L A R tank pressure low.

3. L N 2 tank pressure high.

4. L N 2 tank pressure low.

5. L N 2 tank level low.

6. Compressor air pressure low.

7. Emergency air pressure low.

8. C C tank pressure high.

9. C C tank pressure low.

10. C C I V pressure high.

11. Cold valve pressure low.

12. Temperature device cold.(Trough Temperature)

13. O D H alarm.

14. Air flow device failure. (Ventilation)

15. U P S alarm. (Parameter Alarm)

16. U P S power on. (Inverter On)

17. I $O$ base failure. (Blown Fuse)

18. Air compressor failure. (Off)

19. Air Treatment failure. (Air Drying System)

20. Analog fault. (Transmitter Failure)

21. North tank pressure high.

22. North tank pressure low.

23. North IV pressure high.

24. Detector gas flow low.

25. South tank pressure high.

26. South tank pressure low.

27. South IV pressure high.

28. Building air flow failure.

$29 . \quad$ Unused.

$30 . \quad$ Unused.

31. Unused.

32. Unused. 


\section{DØ AUTO DIALER}

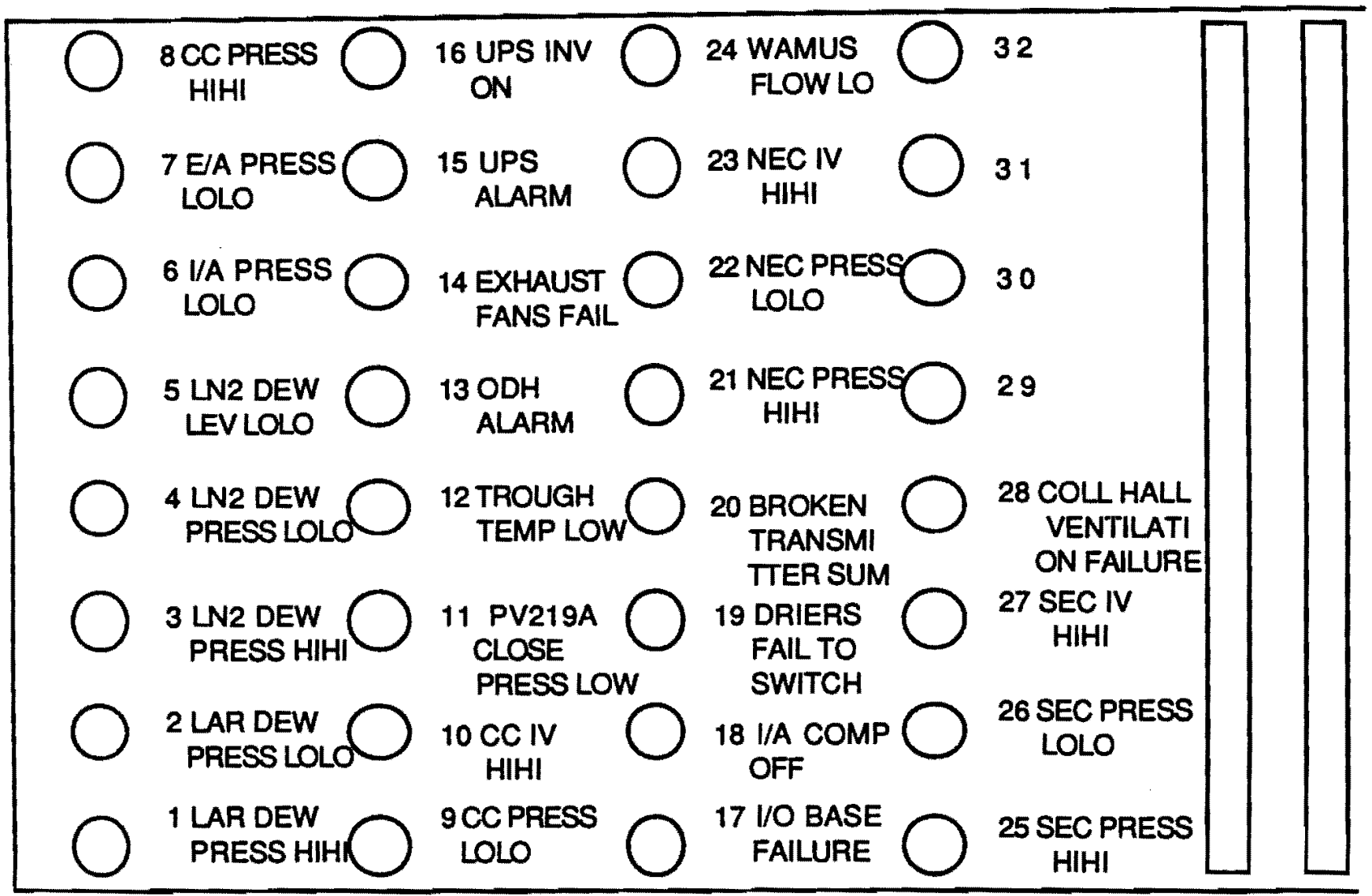

CALL LISTS

CRYO

1) DM PAGE

2) RR PAGE

3) KK PAGE

4) CDF PAGE

5) DM HOME

6) RR HOME

7) KK HOME

8) DO C.R.
CURRENT SETTINGS

WAMUS

1) DØ C.R.

2) CDF PAGE

3) KK PAGE

4) DM PAGE

5) JB HOME

6) RP HOME

7) KK HOME

8)

PRIORITY

1) MESSAGE REPEAT $=2$

2) INTERCALL DELAY $=60 M I N S \quad$ 1) $\mathrm{ODH}$

4) BYPASS TO RUN=6OMINS

5) CALLING HOURS $=24$

6) $\mathrm{ACCESS}$ CODE$=1,2,3,4$

7) POWER FAIL MON=DISABLED

8) TIME BETWEEN CALLS=5MINS
2) CC PRESS HH +LL

2) CC IV PRESS HH

3) ALL THE REST

4) WAMUS GAS SYS

TO ACKNOWLEDGE AN ALARM

1) ON PHONE PUNCH "8" WHEN ASKED FOR ACKNOWLEDGEMENT CR

2) CALL UP BETWEEN OUT CALLS. (840-8065)WHEN THE LINE OR RINGS IT'S ACKNOWLEDGED

3) PUNCH "RESET"(PUTS IN BYPASS)

DONT FORGET TO PUT IN RUN MODE WHEN CONDITION CLEARS. IT WILL AUTOMATICALLY GO BACK TO RUN MODE IN 6OMINS IF YOU DON'T.

\section{CALINCODES}

RUNMODE $=(2,7)$

BYPASS $=(2,2)$

PREMOUS

MODE $=(2,3)$

VERIFY $\quad=(*)$

PROGRAM $=(\#)$

DIAL OUT $=(3)$ 


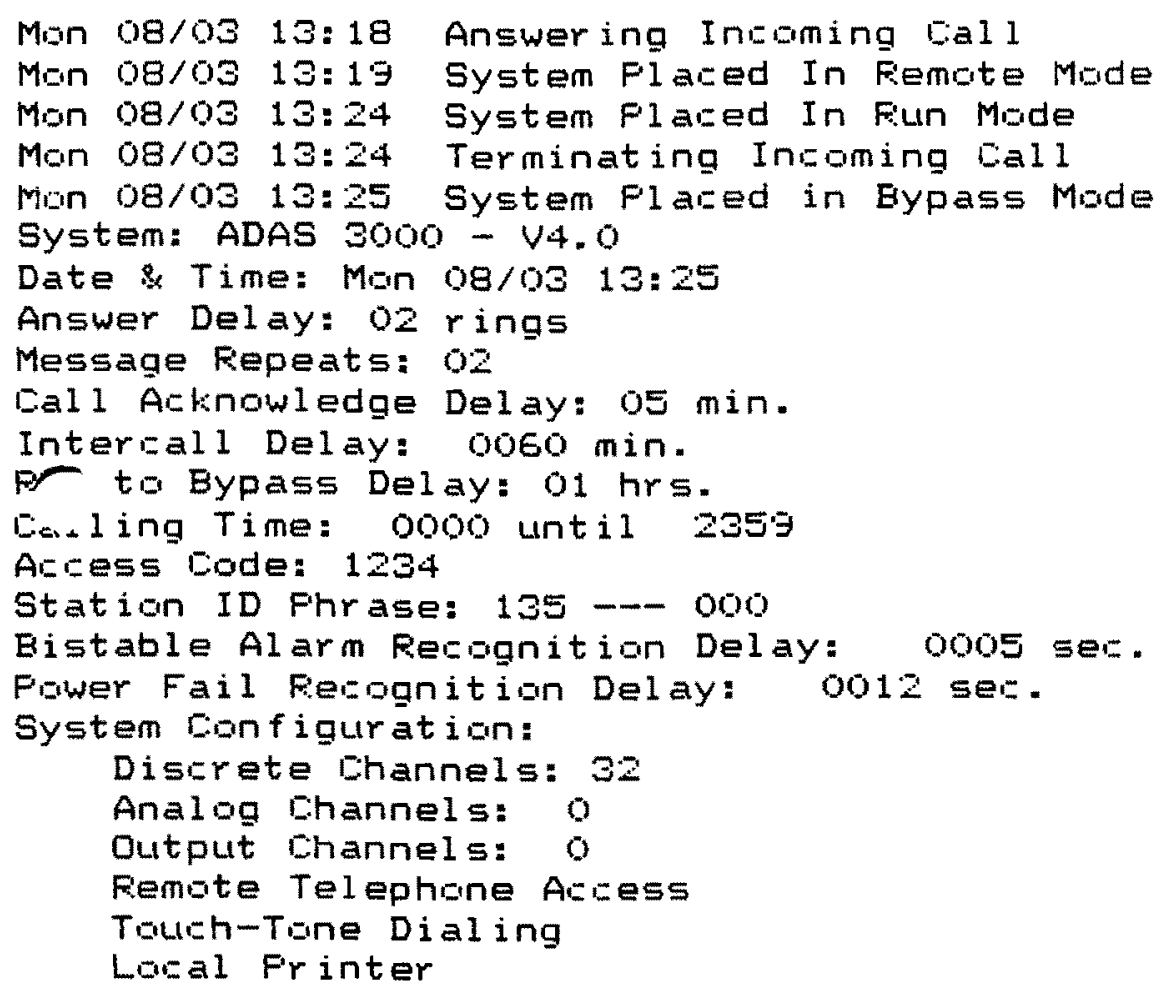




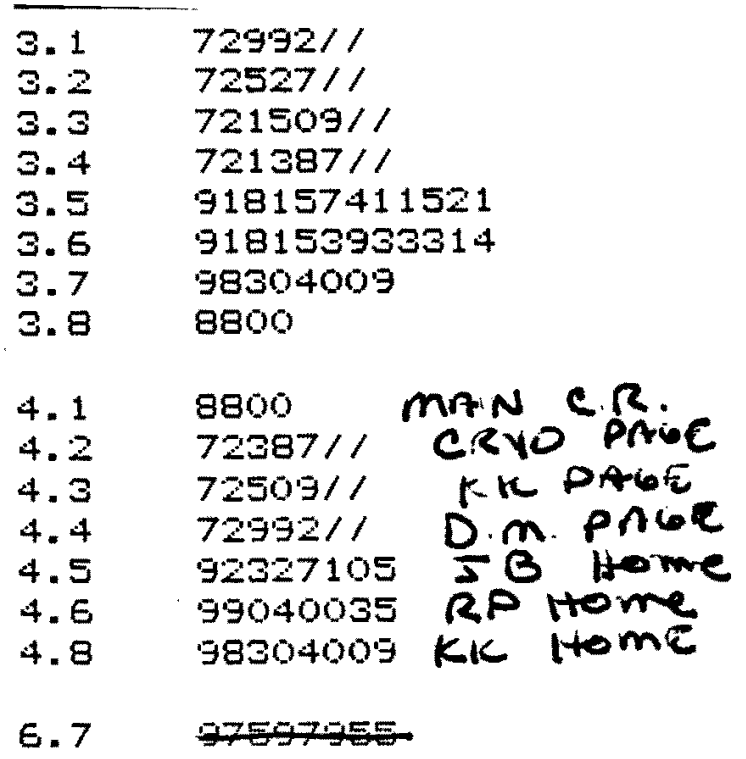

Fower Fail Status

List CD

Bistable Status

\begin{tabular}{|c|c|c|c|c|c|c|c|c|}
\hline \#\# & List & $\mathrm{CD}$ & \multicolumn{2}{|c|}{ Fhr ase } & & & & \\
\hline 1 & $3 A$ & $Y$ & 184 & 095 & 23 & $2 \in 8$ & 225 & 035 \\
\hline 2 & $3 A$ & $\mathrm{Y}$ & 184 & 095 & 233 & 268 & 225 & 043 \\
\hline 3 & $3 A$ & $Y$ & 184 & 204 & 002 & 268 & 225 & 039 \\
\hline 4 & $3 A$ & $Y$ & 184 & 204 & 002 & $2 \in 8$ & 225 & 043 \\
\hline 5 & $3 A$ & $Y$ & 184 & 204 & 002 & 268 & 185 & 043 \\
\hline E & $3 A$ & $Y$ & 129 & 100 & 225 & 043 & & \\
\hline 7 & $3 A$ & Y & 146 & 100 & 225 & 043 & & \\
\hline 8 & $2 A$ & $Y$ & 116 & 116 & 268 & 225 & 039 & \\
\hline 9 & $2 A$ & $Y$ & 116 & 116 & 268 & 225 & 043 & \\
\hline 10 & $2 A$ & $\gamma$ & 116 & 116 & 173 & 282 & 225 & 039 \\
\hline 11 & $3 A$ & $Y$ & 127 & 283 & 225 & 043 & & \\
\hline 12 & $3 A$ & $Y$ & 269 & 070 & 127 & & & \\
\hline 13 & $1 \mathrm{~A}$ & $Y$ & 210 & 135 & 168 & 030 & & \\
\hline 14 & $3 A$ & $Y$ & 100 & 157 & 070 & 154 & & \\
\hline 15 & $3 A$ & $Y$ & 276 & 216 & 245 & 030 & & \\
\hline 16 & $3 A$ & $Y$ & 276 & 216 & 245 & 050 & 076 & \\
\hline 17 & $3 A$ & $Y$ & 173 & 210 & 110 & 154 & & \\
\hline 18 & $3 A$ & $Y$ & 100 & 123 & 154 & & & \\
\hline 19 & $3 A$ & $Y$ & 100 & 273 & 154 & & & \\
\hline 20 & $3 A$ & $\mathrm{Y}$ & 086 & 038 & & & & \\
\hline 21 & $2 A$ & $Y$ & 207 & 268 & 225 & 039 & & \\
\hline 22 & $2 A$ & $Y$ & 207 & 268 & 225 & 043 & & \\
\hline 23 & $2 A$ & $Y$ & 207 & 173 & 282 & 225 & 039 & \\
\hline 24 & $4 A$ & $Y$ & 162 & 157 & 043 & & & \\
\hline 25 & $2 A$ & $Y$ & 261 & 268 & 225 & 037 & & \\
\hline 26 & $2 A$ & $Y$ & $2 \in 1$ & 268 & 225 & 043 & & \\
\hline 27 & $2 A$ & $Y$ & $2 \in 1$ & 173 & 282 & 225 & 039 & \\
\hline 28 & $2 \mathrm{~A}$ & $Y$ & 115 & 100 & 157 & 154 & & \\
\hline 29 & $1 \mathrm{~A}$ & $Y$ & 038 & 020 & 009 & & & \\
\hline 30 & $1 \mathrm{~A}$ & $\mathrm{Y}$ & 038 & 021 & & & & \\
\hline 3 & $1 \mathrm{~A}$ & Y & & & & & & \\
\hline & & $Y$ & & & & & & \\
\hline
\end{tabular}




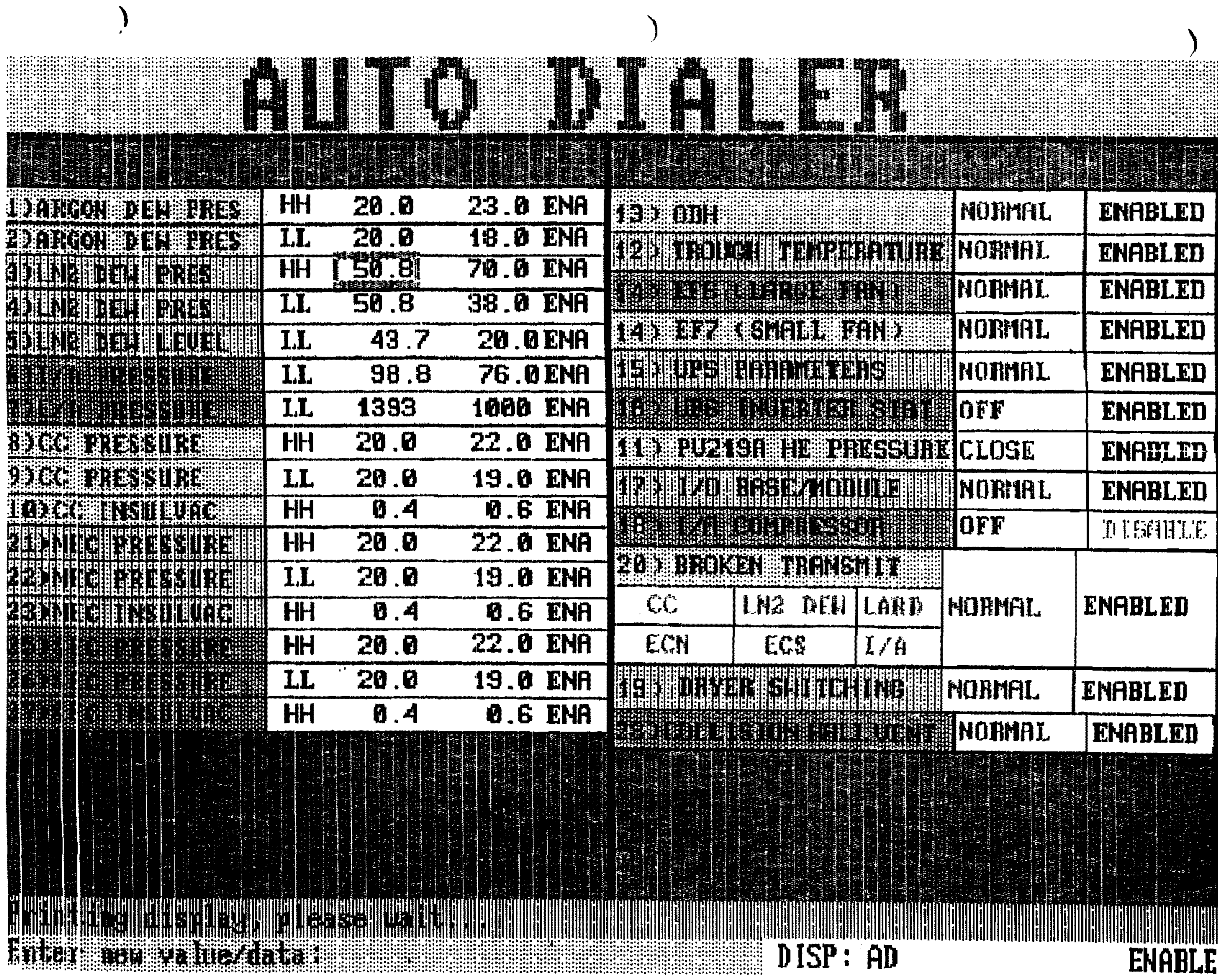


V/O MAP, 7/30/92

\begin{tabular}{|c|c|c|c|c|c|c|c|}
\hline BASE & SLOTIPT & IO TYPE & ADDRESS & TAG & DESCRIPTION & J.B. $\mathbf{B} / \mathbf{C}$ & SEX \\
\hline 1 & $8 / 1$ & RELAYOUT & Y737 & & ODH ALARM - AUTO DIALER CH 13 & U⿴囗十心 & CLSONALARM \\
\hline 1 & $8 / 2$ & RELAYOUT & 738 & & EXHAUST FANS FAIL - AUTO DIALER CH 14 & DiRest & CLSON ALARM \\
\hline 1 & $8 / 3$ & PELAYOUT & 739 & & UPS ALARM - AUTO DIALER CH 15 & 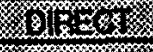 & CLSONALARM \\
\hline 1 & $8 / 4$ & RELAYOUT & 740 & & UPS INVERTER ON - AUTO DIALER CH 16 & B & CLSON ALARM \\
\hline 1 & $8 / 5$ & RELAYOUT & 741 & & BROKEN TRANSMITIER ALARM - AUTO DIALER CH 20 & 0018 & CLS ON ALARM \\
\hline 1 & $8 / 6$ & RELAYOUT & 742 & & DRIEAS FAILED TO SWITCH - AUTO DIALER CH 19 & 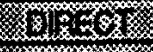 & CLS ON ALARM \\
\hline 1 & $8 / 7$ & PELAYOUT & 743 & & VA COMPRESSOR OFF-AUTO DIALER CH 18 & othes & CLSONALARM \\
\hline 1 & $8 / 8$ & RELAYOUT & 744 & & I/O BASE FAILURE - AUTO DIALER CH 17 & 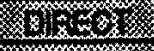 & CLSONALARM \\
\hline 1 & $9 / 1$ & PELAYOUT & Y745 & & CC PRESSURE LOLO-AUTO DIALER CH 9 & Q & CLSONALARM \\
\hline 1 & $9 / 2$ & RELAYOUT & 746 & & CC N HIHI - AUTO DIALER CH 10 & 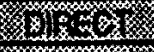 & CLSONALARM \\
\hline 1 & 8/3 & PELAYOUT & 747 & & PV219A CLOSE PRESSURE LOW - AUTO DIALER CH11 & QIIE & CLSONALARM \\
\hline 1 & $9 / 4$ & RELAYOUT & 748 & & TROUGH TEMPERATURE LOW - AUTO DIALER CH 12 & 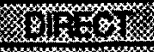 & CLSON ALARM \\
\hline 1 & $9 / 5$ & PELAYOUT & 749 & & PV119A CLOSE PRESSURE LOW - AUTO DIALER CH 24 & Sistin & CLSONALARM \\
\hline 1 & $2 / 6$ & REIAYOUT & 750 & & NEC IV HIHI - AUTO DIALEA CH 23 & 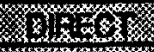 & CLSON ALARM \\
\hline 1 & $9 \sqrt{7}$ & PELAYOUT & 751 & & NEC PRESSURE LOLO-AUTO DIALER CH 22 & 8.1898 & CLSON ALARM \\
\hline 1 & $9 / 8$ & RELAYOUT & 752 & & NEC PRESSURE HIHI - AUTO DIALER CH 21 & 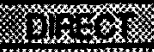 & CLSON ALARM \\
\hline 1 & $10 / 1$ & AO 4-20ma & Y753 & & LN2 DEWARLEVEL LOLO-AUTO DIALEA CH 5 & 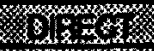 & CLSONALARM \\
\hline 1 & $10 / 2$ & AO 4-20ma & 754 & & VA PRESSURE LOLO - AUTOO DIALER CH 6 & 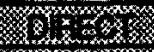 & CLSONALARM \\
\hline 1 & $10 / 3$ & AO 4-20ma & 755 & & EIA PRESSURE LOLO-AUTO DIALER CH 7 & Bing & CLSONALARM \\
\hline 1 & $10 / 4$ & AO 4-20ma & 756 & & CC PRESSURE HIHII - AUTO DIALER CHA & 8 & CLSONALARM \\
\hline 1 & $10 / 5$ & AO 4-20ma & 757 & & SEC PRESSURE HIHI - AUTOO DIALER CH 25 & 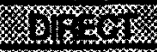 & CLSONALARM \\
\hline 1 & $10 / 6$ & AO 4-20ma & 758 & & SEC PRESSURE LOLO-AUTO DIALER CH 26 & 2. & CLSONALARM \\
\hline 1 & $10 / 7$ & AO 4-20ma & 759 & & SEC IV HIHII - AUTO DIALER CH 27 & 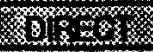 & CLSONALARM \\
\hline 1 & 10/8 & AO $4.20 \mathrm{ma}$ & 760 & & PV319A CLOSE PRESSURE LOW-AUTO DIALER CH 28 & 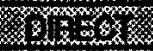 & CLSONALARM \\
\hline 1 & $11 / 1$ & RELAYOUT & Y761 & & LARDEWAR PRESSURE HIHI - AUTO DLALER CH 1 & 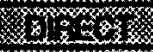 & CLSONALAFM \\
\hline 1 & 11/2 & RELAYOUT & 762 & & LARDEWAR PRESSURE LOLO-AUTODLALERCH 2 & 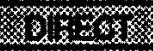 & CLSONALARM \\
\hline 1 & $11 / 3$ & BEIAYOUT & 763 & & LN2 PRESSURE HIIII-AUTO DIALER CH 3 & 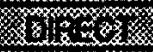 & CLSONALARM \\
\hline 1 & $11 / 4$ & RELAYOUT & 764 & & LN2 PAESSURE LOLO - AUTO DIALER CH 4 & 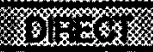 & CLSONALARM \\
\hline
\end{tabular}

